\title{
Anti-inflammatory effect of lycopene on carrageenan-induced paw oedema and hepatic ischaemia-reperfusion in the rat
}

\author{
Letícia Bignotto $^{1}$, João Rocha ${ }^{2}$, Bruno Sepodes ${ }^{2}$, Maria Eduardo-Figueira ${ }^{2}$, Rui Pinto ${ }^{2}$, Marco Chaud ${ }^{3}$, \\ João de Carvalho ${ }^{4}$, Heitor Moreno $\mathrm{Jr}^{1}$ and Helder Mota-Filipe ${ }^{2 *}$ \\ ${ }^{1}$ Departamentos de Farmacologia, Faculdade de Ciências Médicas, Universidade Estadual de Campinas, São Paulo, SP, Brazil \\ ${ }^{2}$ iMED.UL - Inflammation Team, Pharmacological Sciences Group, Unit of Pharmacology and Pharmacotoxicology, Faculty \\ of Pharmacy, University of Lisbon, Avenida das Forças Armadas, 1649-003 Lisboa, Portugal \\ ${ }^{3}$ Faculdade de Ciências da Saúde, Universidade Metodista de Piracicaba, Piracicaba, São Paulo, SP, Brazil \\ ${ }^{4}$ Divisão de Farmacologia, Centro Pluridisciplinar de Pesquisas Químicas, Biológicas e Agrícolas, Universidade Estadual de \\ Campinas, Paulínia, São Paulo, SP, Brazil
}

(Received 10 December 2007 - Revised 16 October 2008 - Accepted 18 October 2008 - First published online 10 February 2009)

\begin{abstract}
The regular intake of tomatoes or its products has been associated with a reduced risk of chronic diseases and these effects have been mainly attributed to lycopene. Here, we evaluated the anti-inflammatory properties of lycopene and its protective effects on organ injury in two experimental models of inflammation. In order to study the effects of lycopene in local inflammation, a carrageenan-induced paw oedema model in rats was performed. Lycopene was administered as an acute $(1,10,25$ or $50 \mathrm{mg} / \mathrm{kg}$, intraperitoneally, $15 \mathrm{~min}$ before carrageenan injection) and chronic treatment $(25$ or $50 \mathrm{mg} / \mathrm{kg}$ per d, $14 \mathrm{~d})$. Inflammation was assessed by the measurement of paw volume increase after $6 \mathrm{~h}$. Lycopene significantly inhibited paw oedema formation at two doses $(25$ and $50 \mathrm{mg} / \mathrm{kg})$ in both acute and repeated administration. The effect of lycopene on liver inflammation was evaluated in a liver ischaemia-reperfusion (I/R) model. Rats were subjected to 45 min of ischaemia of three-quarters of the liver followed by $2 \mathrm{~h}$ of reperfusion. In this model, lycopene was administered daily at two doses $(25 \mathrm{and} 50 \mathrm{mg} / \mathrm{kg})$ during the $14 \mathrm{~d}$ that preceded the experiments. Repeated administration of lycopene reduced liver injury induced by $\mathrm{I} / \mathrm{R}$, as demonstrated by the reduction of the increase in liver injury markers (aspartate aminotransferase, alanine aminotransferase, lactate dehydrogenase and $\gamma$-glutamyl transferase) and attenuation of liver tissue lipoperoxidation was evidenced by a decrease in malondialdehyde production. The present results show that lycopene exhibited local anti-inflammatory activity and also attenuated liver injury induced by I/R. We speculate that lycopene administration might be useful in the pharmacological modulation of inflammatory events.
\end{abstract}

Lycopene: Inflammation: Paw oedema: Ischaemia-reperfusion: Liver

Tomato is one of the most widely consumed fruit/vegetable in the Western civilisation, and it is rich in lycopene and other carotenoids (such as $\alpha-, \beta-, \gamma$-carotene, lutein), potassium, folate, vitamin $\mathrm{C}$ and vitamin $\mathrm{E}^{(1)}$. There is epidemiological data supporting the association between an increased consumption of tomatoes or its products and a reduced risk for developing chronic diseases such as cancer ${ }^{(2,3)}$ and $\mathrm{CVD}^{(1,4,5)}$. These beneficial effects are generally attributed to the antioxidant properties of lycopene ${ }^{(6,7)}$, an acyclic carotenoid with eleven linearly arranged conjugated double bonds ${ }^{(6-9)}$. Lycopene functions as a very potent antioxidant, and this is clearly a major important mechanism of lycopene action. In this regard, lycopene can trap singlet oxygen and reduce mutagenesis in the Ames test ${ }^{(10)}$.

Experimental rodent models suggest that lycopene plays an important role in the modulation of organ injury evoked by inflammatory processes.
The efficacy of lycopene as an anti-inflammatory agent in colitis was evaluated in a rat model of colitis induced by iodoacetamide. This model induces colonic injury due to the production of tissue inflammatory mediators and oxidative radicals. Supplementation of normal diet with lycopene $(200 \mathrm{mg} / \mathrm{kg}$ of normal diet) reduced the histological signs of colon injury, reduced tissue malondialdehyde (MDA) levels and increased superoxide dismutase levels in the red blood cells ${ }^{(11)}$.

The efficacy of lycopene to limit myocardial injury after ischaemia and reperfusion was assessed in a model of 45 min of occlusion of the left anterior descending coronary artery followed by $1 \mathrm{~h}$ of reperfusion in Wistar rats. Daily administration of lycopene $(1 \mathrm{mg} / \mathrm{kg}$, per os (p.o.)) $31 \mathrm{~d}$ before the experiments resulted in a reduction of histological myocardial damage, in the preservation of the myocardial antioxidant status and the inhibition of ischaemia-reperfusion (I/R)-induced lipid peroxidation ${ }^{(12)}$.

Abbreviations: ALT, alanine aminotransferase; AST, aspartate aminotransferase; $\gamma$-GT, $\gamma$-glutamyl transferase; i.p., intraperitoneally; I/R, ischaemia-reperfusion;

MDA, malondialdehyde; p.o., per os; tempol, 4-hydroxy-2,2,6,6-tetramethylpiperidine- $N$-oxyl.

* Corresponding author: Helder Mota-Filipe, fax +351 21795 7458, email hfilipe@ff.ul.pt 
These results suggest that the beneficial effects of lycopene in inflammatory models are probably related to its antioxidant properties.

A vast amount of circumstantial evidence implicates oxygen-derived free radicals (especially superoxide and hydroxyl radical) and potent oxidant substances (such as peroxynitrite) in the I/R injury syndrome, particularly during the reperfusion phase. Generation of free radicals during the reperfusion process can be prevented or attenuated by antioxidants and free radical scavengers ${ }^{(13)}$.

Hence, in the present study, we aimed to evaluate the possible local anti-inflammatory properties of lycopene in acute and repeated administration, and also the effect of repeated administration of lycopene on hepatic injury caused by $I / R$ of the liver.

\section{Material and methods}

\section{Chemicals}

Redivivo $^{\text {TM }}$ (10\% water-soluble lycopene - code 0481041) was obtained from DSM Nutritional Products (São Paulo, SP, Brazil). Redivivo ${ }^{\mathrm{TM}}$ contains $10 \%$ synthetic lycopene finely dispersed in a maize starch-coated matrix of fish gelatin, sucrose and maize oil.

Sodium pentobarbital (Eutasil) was obtained from Sanofi Veterinária (Miraflores, Algés, Portugal). Other compounds were obtained from Sigma-Aldrich Química SA (Sintra, Portugal).

\section{Experimental methods}

Experiments were conducted according to the Home Office Guidance in the Operation of Animals (Scientific Procedures) Act 1986, published by Her Majesty's Stationary Office, London, UK and the Institutional Animal Research Committee Guide for the Care and Use of Laboratory Animals published by the US National Institutes of Health (NIH Publication No. 85-23, revised 1996), as well as to the EC regulations (O.J. of E.C. L 358/1 18/12/1986). Paw oedema studies were carried out using male Wistar rats weighing 100-150 g (Harlan Ibérica, Barcelona, Spain). I/R studies were carried out using male Wistar rats weighing 250$320 \mathrm{~g}$ (Harlan Ibérica, Barcelona, Spain). All animals received a standard diet and water ad libitum.

\section{Paw oedema}

The carrageenan-induced paw oedema of the rat hindpaw is a suitable model to study acute local inflammation and widely considered to be one of the most useful models in the evaluation of anti-inflammatory activity of investigational compounds ${ }^{(14,15)}$.

Paw oedema was induced by a single subplantar injection into the rat left hindpaw of $0.1 \mathrm{ml}$ of a $1 \% \lambda$-carrageenan sterile saline solution. Paw volume was measured by means of a volume displacement method using a plethysmometer (Digital Plethysmometer LE7500; Letica Scientific Instruments, Letica, Spain). Paw volume was measured immediately after the injection of carrageenan ( $V_{0}$ or basal volume) and $6 \mathrm{~h}$ later $\left(V_{6 \mathrm{~h}}\right)$.
Paw oedema was expressed as a percentage of increase in paw volume $6 \mathrm{~h}$ after carrageenan injection relative to the basal values according to the equation:

$$
\%=\frac{V_{6 \mathrm{~h}}-V_{0}}{V_{0}} \times 100 .
$$

The rats were randomly allocated into different groups as described for each experiment.

\section{Paw oedema acute dosing study}

(i) Control group. The animals were subjected to subplantar injection into the rat left hindpaw of $0.1 \mathrm{ml}$ sterile saline and administered with saline $(1 \mathrm{ml} / \mathrm{kg}$, intraperitoneally (i.p.); $n$ 6).

(ii) Carrageenan group. The animals were subjected to paw oedema induction and administered with saline $(1 \mathrm{ml} / \mathrm{kg}$, i.p.; $n 8)$.

(iii) Lycopene1 group. The animals were subjected to paw oedema induction and pretreated with lycopene ( $1 \mathrm{mg} / \mathrm{kg}$, i.p.) $15 \mathrm{~min}$ before $\lambda$-carrageenan injection (n 7).

(iv) Lycopene10 group. The animals were subjected to paw oedema induction and pretreated with lycopene $(10 \mathrm{mg} / \mathrm{kg}$, i.p.) $15 \mathrm{~min}$ before $\lambda$-carrageenan injection $(n 7)$.

(v) Lycopene 25 group. The animals were subjected to paw oedema induction and pretreated with lycopene $(25 \mathrm{mg} /$ $\mathrm{kg}$, i.p.) $15 \mathrm{~min}$ before $\lambda$-carrageenan injection ( $n$ 7).

(vi) Lycopene50 group. The animals were subjected to paw oedema induction and pretreated with lycopene $(50 \mathrm{mg} / \mathrm{kg}$, i.p.) $15 \mathrm{~min}$ before $\lambda$-carrageenan injection ( $n$ 8).

(vii) Trolox group. The animals were subjected to paw oedema induction and pretreated with trolox $(30 \mathrm{mg} / \mathrm{kg}$, i.p.) 15 min before $\lambda$-carrageenan injection $(n 8)$.

(viii) Tempol group. The animals were subjected to paw oedema induction and pretreated with 4-hydroxy2,2,6,6-tetramethylpiperidine- $N$-oxyl (tempol; $30 \mathrm{mg}$ / $\mathrm{kg}$, i.p.) $15 \mathrm{~min}$ before $\lambda$-carrageenan injection $(n 10)$.

(ix) Indomethacin group. The animals were subjected to paw oedema induction and pretreated with indomethacin $(10 \mathrm{mg} / \mathrm{kg}$, i.p. $) 15 \mathrm{~min}$ before $\lambda$-carrageenan injection $(n 8)$.

We have shown in the previous studies with Redivivo $10 \%$ $\mathrm{WS}$, in models of peritonitis in mice, gerbils and rats, that no peritoneal ulceration or mucosal injury is observed in animals administered with this solution i.p. (unpublished results). Also, in the present experiments, no significant changes were observed in polymorphonucleated cell infiltration nor exudates were formed, when Redivivo was administered alone in healthy animals (control group for Redivivo, not shown).

\section{Paw oedema repeated dose study $(14 d)$}

The animals were subjected to daily administration of lycopene by gastric gavage during the $14 \mathrm{~d}$ that preceded the experiments.

(i) Control group. The animals were subjected to subplantar injection into the rat left hindpaw of $0.1 \mathrm{ml}$ sterile saline containing $1 \% \lambda$-carrageenan and administered with saline $(1 \mathrm{ml} / \mathrm{kg}$, p.o.) during $14 \mathrm{~d}$ (n 11). 
(ii) Lycopene 25 group. The animals were subjected to paw oedema induction and treated with lycopene $(25 \mathrm{mg} / \mathrm{kg}$, p.o.) during $14 \mathrm{~d}$ ( $n$ 14).

(iii) Lycopene50 group. The animals were subjected to paw oedema induction and treated with lycopene $(50 \mathrm{mg} / \mathrm{kg}$, p.o.) during $14 \mathrm{~d}$ ( $n$ 14).

\section{Repeated dose toxicity study}

The animals were subjected to daily administration of lycopene by gastric gavage during the $14 \mathrm{~d}$ that preceded the experiments to evaluate possible repeat-dose-associated toxicity.

The rats were randomly assigned to three groups as follows:

(i) Control group. The animals received only water during $14 \mathrm{~d}(n 10)$.

(ii) Lycopene 25 group. The animals were treated with lycopene $(25 \mathrm{mg} / \mathrm{kg}$ per d) during $14 \mathrm{~d}$ ( $n$ 10).

(iii) Lycopene50 group. The animals were treated with lycopene $(50 \mathrm{mg} / \mathrm{kg}$ per $\mathrm{d})$ during $14 \mathrm{~d}$ ( $n$ 10).

At the end of the chronic treatment with lycopene, rats were killed by an overdose of Eutasil and blood was collected by cardiac puncture into a BD Vacutainer SST II Advance gel and clot activator tube (3.5 ml (BD Diagnostics - Prearalytical Systems, Oxford, UK)) and was centrifuged (3000 rpm for $10 \mathrm{~min}$ at room temperature) to separate the serum. The serum was analysed by a local laboratory for clinical chemistry (Clinica Medica e Diagnostico Dr Joaquim Chaves, Miraflores, Alges, Portugal). Alanine aminotransferase (ALT, a specific marker for hepatic parenchymal injury) and aspartate aminotransferase (AST, a non-specific marker for hepatic injury) were measured to evaluate the liver function. Lactate dehydrogenase (an unspecific cellular injury marker) was measured to evaluate the general organic function. Amylase was measured to evaluate the pancreatic function. Urea and creatinine were measured to evaluate the renal function.

\section{Liver ischaemia/reperfusion study}

The liver, and, in particular, hepatic cells, plays a central role in the metabolism of several compounds such as vitamins, lipids, drugs, etc. I/R liver injury occurs in several clinical and pathological situations and consists of an interruption of blood supply to the liver followed by reperfusion. When the blood supply is restored, the organs are usually subjected to a further insult, aggravating the injury created within the ischaemic period ${ }^{(16,17)}$. This reperfusion insult is a direct consequence of a complex interplay between different mechanisms. At the beginning of liver reperfusion, microcirculation failure results from endothelial cell activation, vasoconstriction, leucocyte recruitment and entrapment (leukostasis), and platelet aggregation within the sinusoids ${ }^{(18)}$. These effects prolong hypoxia, since the areas of the liver remain ischaemic despite the onset of reperfusion, resulting in the activation of Kupffer cells and neutrophils, which produce pro-inflammatory cytokines and oxygen-derived free radicals, further aggravating the hepatic injury $(19,20)$

For these experiments, all rats were anaesthetised with Eutasil $(60 \mathrm{mg} / \mathrm{kg}$, i.p., Sanofi Veterinaria) and anaesthesia was maintained by supplementary i.p. boli of Eutasil.
Briefly, anaesthetised rats were placed onto a thermostatically controlled heating mat (Harvard Apparatus Ltd, Kent, UK) and body temperature maintained at $37 \pm 0.5^{\circ} \mathrm{C}$ by means of a rectal probe attached to a homoeothermic blanket. A tracheotomy was performed to maintain airway patency and to facilitate spontaneous respiration. The jugular vein was cannulated (PP25, internal diameter $0.40 \mathrm{~mm}$, Portex, Hythe, Kent, $\mathrm{UK}$ ) for the administration of saline or anaesthesia as required. A midline laparotomy was performed in order to carefully expose the liver.

The rats were randomly allocated into five groups as follows:

(i) Control group. The data were obtained from nonmanipulated animals, i.e. rats which were not subjected to any surgical procedure ( $n$ 10).

(ii) Sham group. Rats which were subjected to the surgical procedures described later except for liver I/R. Rats which received vehicle as a daily administration by gastric gavage during the $14 \mathrm{~d}$ that preceded the experiments to liver I/R, and maintained under anaesthesia for the duration of the experiment ( $n$ 11).

(iii) $I / R$ group. Rats which were subjected to the surgical procedures described later and underwent liver ischaemia for $45 \mathrm{~min}$ followed by reperfusion for $2 \mathrm{~h}(n 8)$.

(iv) $I / R+$ lycopene group. Rats which received lycopene $(25 \mathrm{mg} / \mathrm{kg}$ per d) as a daily administration by gastric gavage during the $14 \mathrm{~d}$ that preceded the experiments and underwent the full I/R procedure $(n 7)$.

(v) Lycopene group. Rats which received lycopene $(25 \mathrm{mg} / \mathrm{kg}$ per d) as a daily administration by gastric gavage during the $14 \mathrm{~d}$ that preceded the experiments and underwent the surgical procedures described for the sham group ( $n$ 14).

For the liver I/R surgical procedures, ligament attachments connecting the liver, diaphragm, abdominal wall and neighbouring organs were divided. The liver hilus was exposed to find the common hepatic artery and the portal vein. A vascular microclamp was used to interrupt the portal venous and arterial hepatic blood supply to the three cephalic lobes of the liver during $45 \mathrm{~min}$. The three caudal lobes retained an intact portal inflow and venous outflow, preventing the intestinal venous congestion and possible leakage of bacteria or bacterial products to the circulation. Reperfusion commenced once the vascular clip was removed, and was allowed to proceed for $2 \mathrm{~h}$. Occlusion was verified visually by the change in the colour of the liver to a paler shade, and reperfusion by a blush. Other rats were subjected to sham operation (shamoperated), which underwent surgical procedures similar to the I/R group rats but did not undergo I/R of the liver clamping and were maintained under anaesthesia for the duration of the experiment. At the end of all experiments, rats were killed by an overdose of an anaesthetic.

\section{Measurement of biochemical parameters}

At the end of the reperfusion period for the liver I/R experiments and whenever appropriate regarding other experiments, blood was collected into a serum SST gel and clot activator tube (Becton, Dickinson and Company) and was centrifuged (3000 rpm for $10 \mathrm{~min}$ at room temperature) to separate 
the serum. The serum was analysed within $24 \mathrm{~h}$ by a laboratory for clinical chemistry (Clinica Medica e Diagnostico Dr Joaquim Chaves). Liver injury was assessed by measuring the rise in the serum levels of ALT (a specific marker for hepatic parenchymal injury), AST (a non-specific marker for hepatic injury) and $\gamma$-glutamyl transferase $(\gamma$-GT, a very sensitive but non-specific indicator of liver disease with the increased activity found in both the cholestasis and hepatocellular damage).

\section{Measurement of liver tissue malondialdehyde}

MDA levels in the liver tissue were determined as an indicator of lipid peroxidation. Briefly, liver tissues, collected at the end of the I/R experiment, were homogenised in $1.15 \% \mathrm{KCl}$ solution. An aliquot $(100 \mu \mathrm{l})$ of the homogenate was added to a reaction mixture containing $200 \mu \mathrm{l}$ of $8.1 \%$ (w/v v/v) SDS, $1500 \mu \mathrm{l}$ of $20 \%(\mathrm{v} / \mathrm{v})$ acetic acid $(\mathrm{pH} 3 \cdot 5), 1500 \mu \mathrm{l}$ of $0.08 \%(\mathrm{w} / \mathrm{v})$ thiobarbituric acid and $700 \mu \mathrm{l}$ distilled water. The samples were then boiled for $1 \mathrm{~h}$ at $95^{\circ} \mathrm{C}$ and centrifugated at $3000 \mathrm{~g}$ for $10 \mathrm{~min}$. The absorbance of the supernatant was measured by spectrophotometry at $650 \mathrm{~nm}$.

\section{Measurement of antioxidant capacity}

The method for determining the antioxidant capacity of the extract used was the oxygen radical absorbance capacity method described by $\mathrm{Ou}$ et al. ${ }^{(21)}$. This method is based on the ability of certain substances to inhibit fluorescein oxidation induced by peroxyl radicals (ROO) generated by $2^{\prime}, 2^{\prime}$-azobis(2-amidinopropane)dihydrochloride. The assay consists of the addition of $300 \mu \mathrm{l}$ of the sample to a fluorescein solution $\left(1800 \mu \mathrm{l}, 4 \times 10^{-6} \mathrm{mM}\right)$ at $37^{\circ} \mathrm{C}$. After $2^{\prime}, 2^{\prime}$-azobis (2-amidinopropane)dihydrochloride addition, the fluorescence of the mixture is measured every minute, during $30 \mathrm{~min}$ $\left(\lambda_{\mathrm{em}}=515\right.$ and $\left.493 \mathrm{~nm}\right)$. All solutions are prepared in PBS (75 mM, pH 7.4). Antioxidation values are calculated through the loss of fluorescein fluorescence during the $30 \mathrm{~min}$ by comparison with an antioxidant standard, trolox. The final result is expressed in terms of trolox equivalents.

\section{Statistical analysis}

The results were expressed as the means with their standard errors and were compared using a one-factorial ANOVA test, followed by a Dunnett's post hoc test. A $P$ value less than 0.05 was considered to be statistically significant.

\section{Results}

Paw oedema acute dosing study

Intraplantar injection of carrageenan in rats led to an increase in paw volume after $6 \mathrm{~h}(46.9$ (SEM 7.6)) \% when compared with the control group (Fig. 1). This increase was significantly reduced in a dose-dependent manner by pretreatment with lycopene, with the most significant effect observed at the doses 25 and $50 \mathrm{mg} / \mathrm{kg}$.

A comparison of the effect of a single administration of lycopene (25 and $50 \mathrm{mg} / \mathrm{kg}$ ) with the effect of tempol

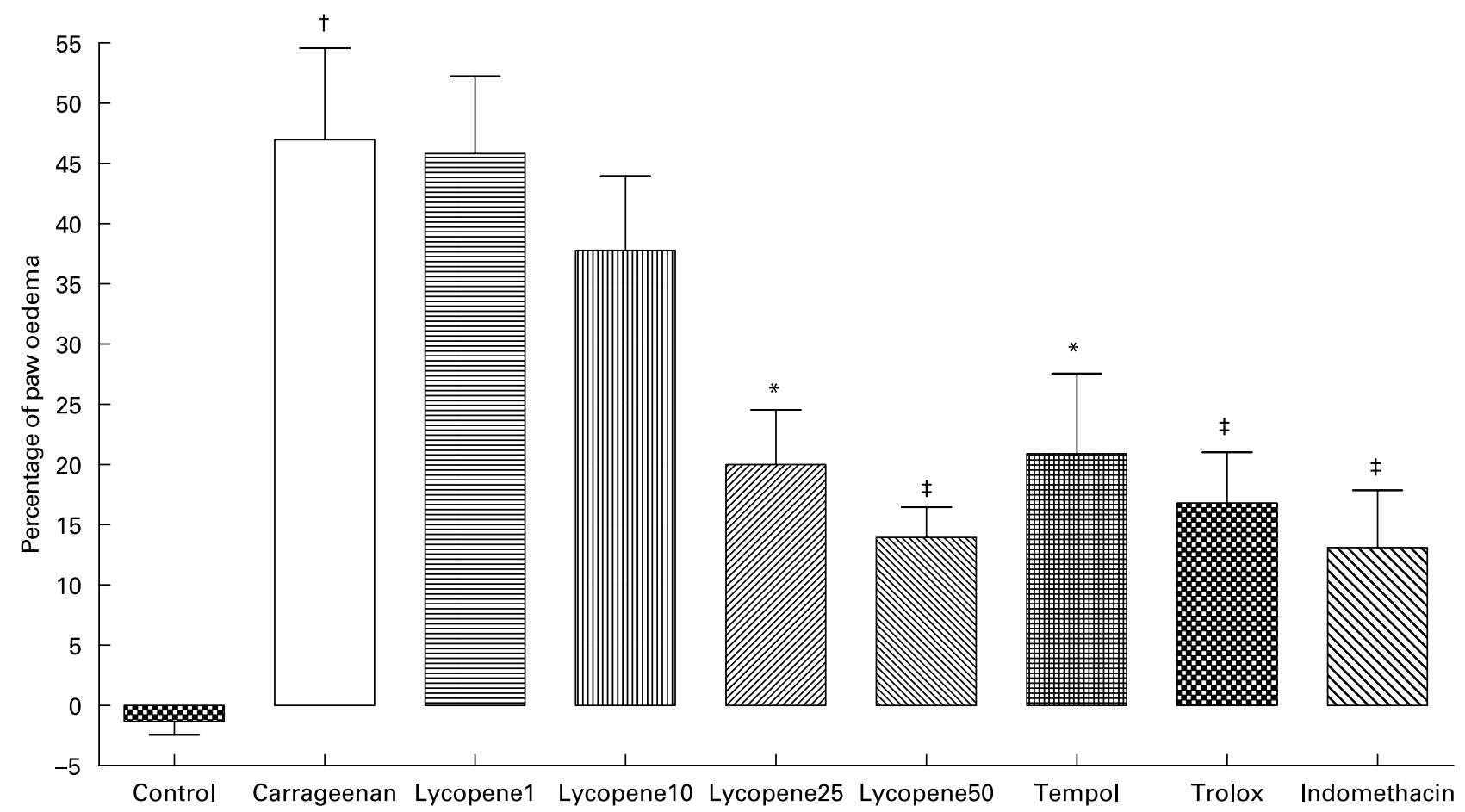

Fig. 1. Effect of a single administration of lycopene (1 mg/kg, intraperitoneally (i.p.; $n$ 7), $10 \mathrm{mg} / \mathrm{kg}$, i.p. $(n$ 7), $25 \mathrm{mg} / \mathrm{kg}$, i.p. $(n 7)$ and $50 \mathrm{mg} / \mathrm{kg}$, i.p. $(n$ 8)) on rat paw oedema development elicited by carrageenan $6 \mathrm{~h}$ after oedema induction and comparison with the effect of tempol $(30 \mathrm{mg} / \mathrm{kg}$, i.p.; $n$ 10), trolox (30 $\mathrm{mg} / \mathrm{kg}$, i.p.; $n$ 8) and indomethacin ( $10 \mathrm{mg} / \mathrm{kg}$, i.p.; $n$ 8). Lycopene administered at 25 and $50 \mathrm{mg} / \mathrm{kg}$ significantly inhibited rat paw oedema formation and the reduction was in the same magnitude as tempol, trolox and indomethacin. The data are presented as means with their standard errors. ${ }^{\star} P<0.05 v$. carrageenan group; $\dagger P<0.01 v$. control group; $\ddagger P<0.01 v$. carrageenan group. 
$(30 \mathrm{mg} / \mathrm{kg})$, trolox $(30 \mathrm{mg} / \mathrm{kg})$ and indomethacin $(10 \mathrm{mg} / \mathrm{kg})$ showed that the lycopene administration reduced oedema formation in the same magnitude as tempol, trolox and indomethacin (Fig. 1).

\section{Paw oedema repeated dose study}

Carrageenan-induced oedema was significantly reduced in the animals treated daily with lycopene at the doses 25 and $50 \mathrm{mg} / \mathrm{kg}$ for $14 \mathrm{~d}$ (Fig. 2). The reduction of carrageenaninduced oedema was not significantly different between the doses 25 and $50 \mathrm{mg} / \mathrm{kg}$.

\section{Repeated dose toxicity study $(14 d)$}

Daily administration of lycopene during $14 \mathrm{~d}$ at the doses of 25 and $50 \mathrm{mg} / \mathrm{kg}$ by oral gavage did not exhibit any significant differences in ALT, AST, lactate dehydrogenase, amylase, urea and creatinine serum levels when compared with the control group (Table 1).

\section{Effect of lycopene treatment on liver injury}

In sham-operated rats, the surgical procedure did not produce a significant change in the serum levels of AST (Fig. 3) and ALT (Fig. 3) when compared with the control group. When compared with sham-operated rats, the I/R of the liver resulted in significant rises in the serum levels of the marker enzymes AST, ALT and $\gamma$-GT, demonstrating the development of hepatocellular injury. In rats subjected to I/R pretreated with lycopene, no significant rise in the $\gamma$-GT serum levels was detected when compared with the sham group (Fig. 4). Significant rises in the serum levels of the enzymes AST and ALT were observed in the group pretreated with lycopene $+\mathrm{I} / \mathrm{R}$ when compared with the sham group, but the serum levels of these marker enzymes were lower than the I/R group (Fig. 3).

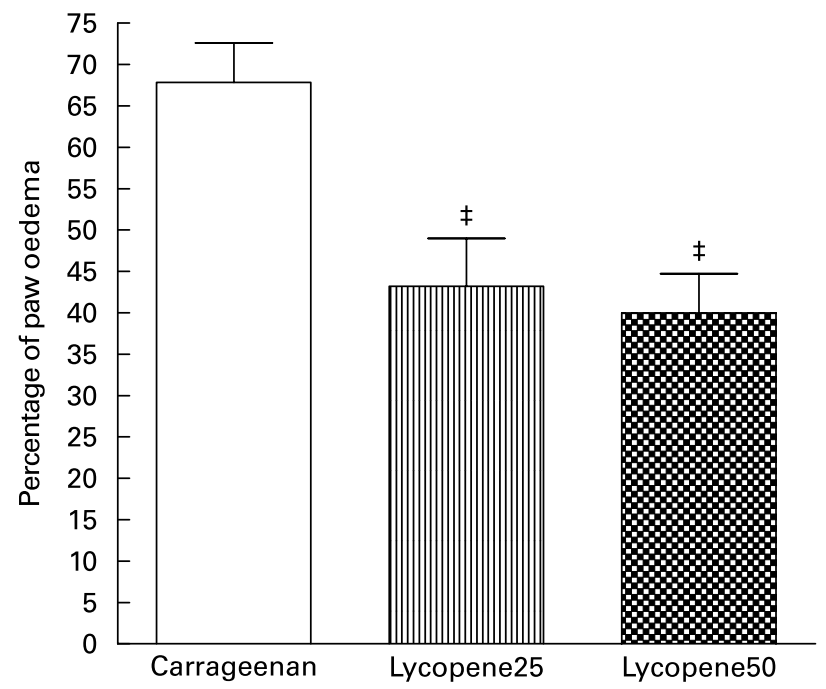

Fig. 2. Effect of lycopene administered daily (14 d) by gastric gavage at two doses $(25 \mathrm{mg} / \mathrm{kg}$ per d; $n 14$ or $50 \mathrm{mg} / \mathrm{kg}$, per os; $n$ 14) on rat paw oedema development elicited by carrageenan. Lycopene administered at 25 and $50 \mathrm{mg} / \mathrm{kg}$ significantly inhibited rat paw oedema formation. There was no statistical differences between the effect of both doses. The data are presented as means with their standard errors. $\ddagger P<0.01 \mathrm{v}$. carrageenan group.
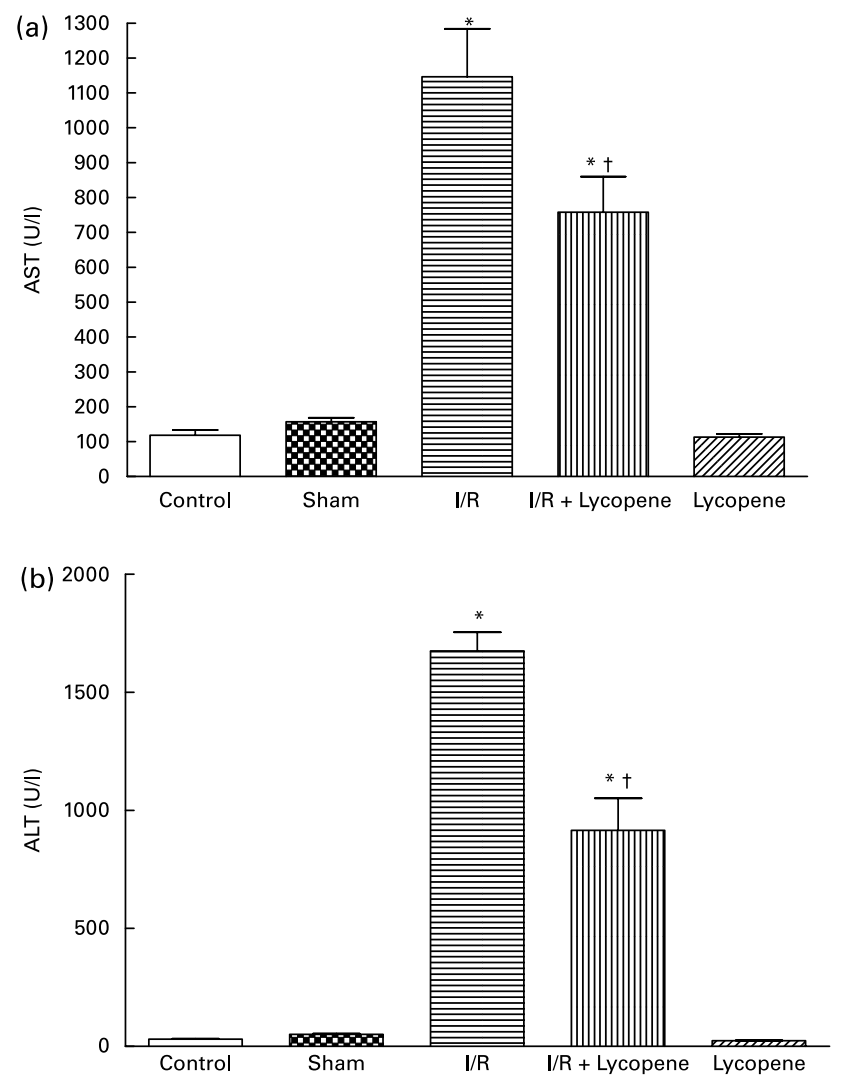

Fig. 3. Effect of lycopene on (a) aspartate aminotransferase (AST) and (b) alanine aminotransferase (ALT) levels in the serum. When compared with sham-operated animals, there was a significant rise in the serum levels of AST and ALT in the group subjected only to liver ischaemia-reperfusion $(\mathrm{I} / \mathrm{R})$. In both cases, daily administration of lycopene during the $14 \mathrm{~d}$ that preceded the experiments significantly reduced this increase. Each value is the means with their standard errors for $n$ animals (control group, $n 10$; sham group, $n 11$; I/R group, $n 8$; I/R + lycopene group, $n 7$; lycopene group, $n 14$ ). ${ }^{\star} P<0.01 \mathrm{v}$. sham group; $\dagger P<0.01 \mathrm{v}$. I/R group.

Lycopene, by itself, when administered to rats not subjected to I/R injury, had no significant effect on the AST and ALT levels (Fig. 3).

\section{Effect of lycopene treatment on the liver tissue levels of malondialdehyde}

Pretreatment with lycopene resulted in a decrease in the liver MDA levels when compared with the liver samples from the $\mathrm{I} / \mathrm{R}$ group. This indicates that lipid peroxidation of the liver tissue was attenuated on the pretreated organs subjected to $\mathrm{I} / \mathrm{R}$ (Fig. 5).

\section{Antioxidant capacity}

The antioxidant capacity of lycopene, calculated as trolox equivalents, was 1867 (SEM 78.6) $\mu \mathrm{mol}$ trolox equivalents/g.

\section{Discussion}

The production of reactive oxygen species and an imbalance of the cellular oxidative status have been related to the initiation and progression of inflammatory processes, and 
Table 1. Effects of lycopene administration during the $14 \mathrm{~d}$ by gastric gavage at two doses $(25 \mathrm{or} 50 \mathrm{mg} / \mathrm{kg}$, per os (p.o.)) on the plasma concentration of alanine aminotransferase (ALT), aspartate aminotransferase (AST), lactate dehydrogenase (LDH), amylase, urea and creatinine

(Mean values with their standard errors)

\begin{tabular}{|c|c|c|c|c|c|c|c|c|c|c|c|c|}
\hline & \multicolumn{2}{|c|}{ ALT (U/I) } & \multicolumn{2}{|c|}{ AST (U/I) } & \multicolumn{2}{|c|}{ LDH (U/I) } & \multicolumn{2}{|c|}{ Amylase (U/l) } & \multicolumn{2}{|c|}{ Urea (mg/l) } & \multicolumn{2}{|c|}{$\begin{array}{l}\text { Creatinine } \\
(\mathrm{mg} / \mathrm{l})\end{array}$} \\
\hline & Mean & SE & Mean & SE & Mean & SE & Mean & SE & Mean & SE & Mean & SE \\
\hline Control $(n 10)$ & $30 \cdot 80$ & 1.7 & $118 \cdot 70$ & $15 \cdot 1$ & $503 \cdot 50$ & $105 \cdot 8$ & $1932 \cdot 40$ & $97 \cdot 1$ & 334.0 & 8 & $2 \cdot 4$ & 0.1 \\
\hline $\begin{array}{l}\text { Lycopene }(25 \mathrm{mg} / \mathrm{kg} \text {, } \\
\text { p.o. }(n 10))\end{array}$ & $24 \cdot 40$ & 1.5 & $115 \cdot 20$ & $13 \cdot 3$ & $719 \cdot 70$ & 109.6 & $1920 \cdot 50$ & $137 \cdot 8$ & $288 \cdot 0$ & 6 & 2.5 & 0.1 \\
\hline $\begin{array}{l}\text { Lycopene }(50 \mathrm{mg} / \mathrm{kg} \text {, } \\
\text { p.o. }(n 10))\end{array}$ & $25 \cdot 70$ & 3.4 & $105 \cdot 30$ & $7 \cdot 4$ & 482.50 & $51 \cdot 3$ & $1791 \cdot 00$ & 98.0 & $314 \cdot 0$ & 16 & $2 \cdot 2$ & 0.1 \\
\hline
\end{tabular}

Blood was collected at the 15 th day and centrifuged at $3000 \mathrm{rpm}$ for $10 \mathrm{~min}$ to obtain the serum. Statistical analysis did not reveal any significant differences between the lycopene and control groups.

the administration of antioxidant substances has proven to be useful in the reduction of injury associated with several situations of shock, inflammation and I/R injury ${ }^{(22)}$.

Lycopene is a carotenoid with known antioxidant properties, and as re-evaluated by the oxygen radical absorbance capacity method in the present paper, it revealed a high antioxidant capacity (1867 (SEM 78.6) $\mu$ mol trolox equivalents/g).

Thus, knowing the importance of antioxidant substances on inflammation situations, we aimed to evaluate the anti-inflammatory activity of lycopene. Therefore, we evaluated the local anti-inflammatory properties of lycopene on a model of carrageenan-induced paw oedema, when acutely and chronically administered, and also the effect of chronically administered lycopene in a model of hepatic injury caused by the I/R of the liver.

The results show that lycopene administration significantly reduced oedema formation $6 \mathrm{~h}$ after the induction by intraplantar injection of carrageenan, thus exhibiting a local antiinflammatory effect. This effect was observed when the administration was performed immediately before induction or by repeated administration during the $14 \mathrm{~d}$ that preceded the experiment. In the acute dosing study, a dose-response relationship was observed, achieving a maximal effect of $30 \%$ of oedema reduction at the $50 \mathrm{mg} / \mathrm{kg}$ dose. In the repeated dosing study, both the doses evaluated revealed

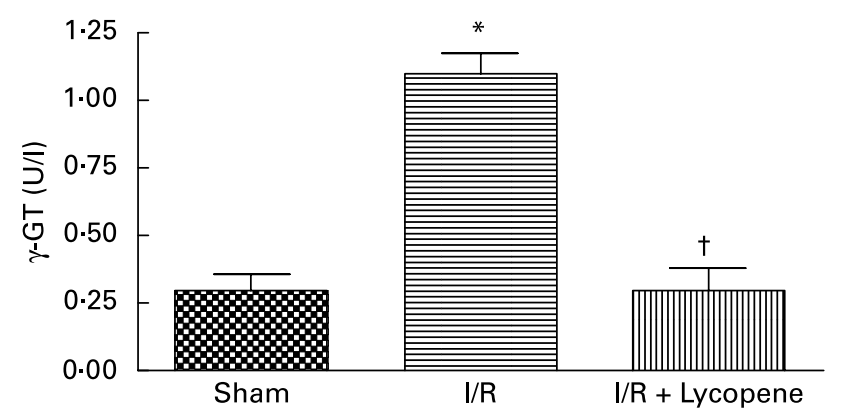

Fig. 4. Effect of lycopene treatment on $\gamma$-glutamyl transferase $(\gamma-G T)$ serum levels. When compared with sham-operated animals, there was a significant rise in the serum levels of $\gamma$-GT in the group subjected only to liver ischaemia-reperfusion (I/R). Daily administration of lycopene during the $14 \mathrm{~d}$ that preceded the experiments significantly reduced this increase in $\gamma$-GT serum levels with no significant difference from sham-operated rats. Each value is the means with their standard errors for $n$ animals (sham group, $n 11 ; \mathrm{I} / \mathrm{R}$ group, $n$ 8; I/R group + Lycopene, $n$ 7). ${ }^{*} P<0.01 \mathrm{v}$. sham group; $\dagger P<0.01$ v. I/R group.
$30 \%$ of oedema reduction with no dose-response relationship. This probably reflects that a maximal anti-inflammatory effect is obtained when lycopene is administered at the tested doses during $14 \mathrm{~d}$.

This local acute inflammation model induces a biphasic oedema consisting of an early phase (up to $2 \mathrm{~h}$ ) followed by a more sustained late phase $(2-6 \mathrm{~h})$. The early phase of carrageenan oedema is related to the production of immediate inflammation mediators such as histamine, bradykinin, leukotrienes, platelet-activating factor and cyclo-oxygenase products in the inflamed tissue. The late phase is related to neutrophil infiltration ${ }^{(23)}$ and the production of reactive species such as hydrogen peroxide, superoxide radical, peroxinitrite $^{(24)}$ and pro-inflammatory prostanoids ${ }^{(25)}$.

Giving the importance of the oxidative status in the formation of oedema, the anti-inflammatory effect exhibited by lycopene in this model might be related to its antioxidant properties.

Lycopene also exhibited the anti-inflammatory effect in a rat model of liver injury induced by $\mathrm{I} / \mathrm{R}$ in which the administration of lycopene during $14 \mathrm{~d}$ led to a reduction of liver injury. The increase in the serum levels of AST and ALT

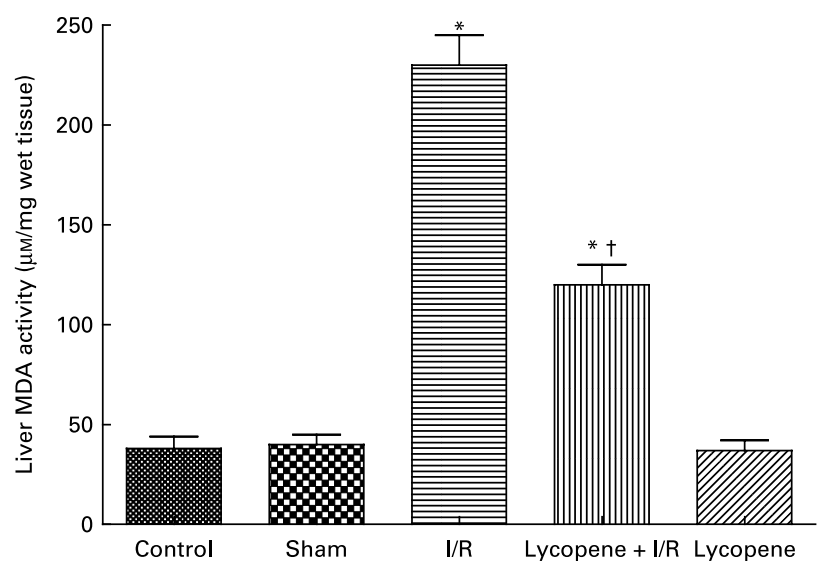

Fig. 5. Effect of lycopene treatment on the liver tissue levels of malondialdehyde (MDA). Pretreatment with lycopene resulted in a decrease in the liver MDA levels when compared with the liver samples from the ischaemiareperfusion $(\mathrm{I} / \mathrm{R})$ group. This indicates that lipid peroxidation of the liver tissue was reduced on the pretreated organs subjected to I/R. Each value is the means with their standard errors for $n$ animals (control group, $n 4$; sham group, $n$ 9; I/R group, $n$ 7; lycopene + I/R group, $n$ 7; lycopene group, $n$ 4). ${ }^{\star} P<0.001 \mathrm{v}$. sham group; $\dagger P<0.001 \mathrm{v}$. l/R group. 
induced by $\mathrm{I} / \mathrm{R}$ was significantly attenuated by pretreatment with lycopene and the $\gamma$-GT serum levels in lycopene-treated rats were not significantly different from sham-operated rats (not subjected to I/R).

Measurement of MDA levels in the liver tissue revealed that I/R injury increases MDA production, a consequence of an increase in lipid peroxidation. Liver samples from lycopenetreated rats exhibited lower levels of tissue MDA than rats subjected only to $\mathrm{I} / \mathrm{R}$. This indicates that lipid peroxidation of the liver tissue was reduced on the pretreated organs subjected to I/R.

This suggests that the beneficial effect of lycopene on the reduction of liver injury might be related to its antioxidant properties.

I/R injury occurs in a number of clinical settings, including liver surgery, transplantation and haemorrhagic shock with subsequent fluid resuscitation, leading to significant morbidity and mortality. The mechanism underlying I/R damage to liver is likely to be a multifactor phenomenon involving a complex interaction between different mechanisms such as endothelial cell activation, vasoconstriction, the activation of Kupffer cells and neutrophils that produce pro-inflammatory mediators such as reactive species. The reactive species produced in liver $\mathrm{I} / \mathrm{R}$ are one of the elements responsible for the worsening of cellular damage found in this model ${ }^{(19,26)}$. The administration of antioxidant therapy has been proposed as a new pharmacological tool in the reduction of the damage caused by reactive species in I/R situations ${ }^{(27)}$. Sepodes et al. ${ }^{(13)}$ confirmed this beneficial antioxidant effect using tempol, an intracellular free radical scavenger. In that study, tempol limited the damage provoked by liver I/R injury, improving liver function and resistance to injury.

The improvement on hepatic function markers in $I / R$ conditions by lycopene is in agreement with the other $I / R$ damage model. In the myocardial I/R model, the early intervention with lycopene resulted in the preservation of the myocardial antioxidant status; it reduced the lipid peroxidation induced by this model and produced a reduction of myocardial damage $^{(12)}$.

We also evaluated the systemic toxicity of lycopene at the doses of $25 \mathrm{mg} / \mathrm{kg}$ per $\mathrm{d}$ and $50 \mathrm{mg} / \mathrm{kg}$ per d during $14 \mathrm{~d}$. Serum levels of biomarkers for organ injury were evaluated. No significant difference was observed between the lycopene-treated and control groups, indicating that lycopene administration during $14 \mathrm{~d}$ did not lead to kidney, liver or pancreatic injury (Table 1). This is in agreement with other studies in which lycopene was considered to have a relatively large margin of safety with an absence of toxic effects in the long-term administration of higher doses ${ }^{(28)}$.

In conclusion, the present results show, for the first time, that lycopene exhibits local anti-inflammatory activity in the carrageenan-induced paw oedema model and attenuates liver injury induced by $\mathrm{I} / \mathrm{R}$, which appears to be related to its antioxidant properties.

Therefore, lycopene administration might be useful in the management of inflammatory processes and further research on anti-inflammatory carotenoids might lead to the discovery of new pharmacological tools in the treatment of inflammatory diseases.

To conclude, the present study aims to show that, apart from its nutritional character, lycopene showed anti-inflammatory activity and reduced liver injury induced by $\mathrm{I} / \mathrm{R}$. This nutrient might be useful in the prophylaxis of current damage caused by inflammatory processes, and this protection is evident when lycopene is administrated chronically, suggesting that the regular consumption of lycopene-rich products (such as tomatoes and their products) might help to limit inflammatory damages and improve health.

\section{Acknowledgements}

We thank Cláudia Fernandes for the outstanding assistance during the present study, DSM Nutritional Products for supplying Redivivo $^{\mathrm{TM}}$, Clinica Medica e Diagnóstico, Dr Joaquim Chaves for the remarkable technical assistance during the study. L. B. was supported by a grant from Coordenação de Aperfeiçoamento de Pessoal de Nível Superior. There is no conflict of interest. L. B., J. R. and B. S. contributed equally to the findings reported in the paper. L. B. and J. R. contributed to the study design, data collection and interpretation, statistical analysis, manuscript preparation and literature search. B. S. contributed to the study design, data collection and interpretation, statistical analysis, manuscript preparation and fund collection. M. E.-F. contributed to the data collection, data interpretation and manuscript preparation. R. P. contributed to the data collection. M. C., J. C. and H. M. contributed to the data interpretation and fund collection. H. M.-F. contributed to the study design, data interpretation, manuscript preparation, literature search and fund collection.

\section{References}

1. Canene-Adams K, Campbell JK, Zaripheh S, et al. (2005) The tomato as a functional food. J Nutr 135, 1226-1230.

2. Giovannucci E, Ascherio A, Rimm EB, et al. (1995) Intake of carotenoids and retinol in relation to risk of prostate cancer. $J$ Natl Cancer Inst 87, 1767-1776.

3. Giovannucci E (2005) Tomato products, lycopene, and prostate cancer: a review of the epidemiological literature. $J$ Nutr $\mathbf{1 3 5}$, 2030S-2031S.

4. Blum A, Monir M, Wirsansky I, et al. (2005) The beneficial effects of tomatoes. Eur J Intern Med 16, 402-404.

5. Saganuma H \& Inakuma T (1999) Protective effect of dietary tomato against endothelial dysfunction in hypercholesterolemic mice. Biosci Biotechnol Biochem 63, 78-82.

6. Clinton SK (1998) Lycopene: chemistry, biology and implications for human health and disease. Nutr Rev 56, 35-51.

7. Arab L \& Steck S (2000) Lycopene and cardiovascular disease. Am J Clin Nutr 71, Suppl. 6, S1691-S1695.

8. Di Mascio P, Kaiser S \& Sies H (1989) Lycopene as the most efficient biological carotenoid singlet oxygen quencher. Arch Biochem Biophys 274, 532-538.

9. Sies H \& Stahl W (1995) Vitamins E and C, beta-carotene, and other carotenoids as antioxidants. Am J Clin Nutr 62, 1315S-1321S.

10. Heber D \& Lu QY (2002) Overview of mechanisms of action of lycopene. Exp Biol Med (Maywood) 227, 920-923.

11. Reifen R, Nissenkorn A, Matas Z, et al. (2004) 5-ASA and lycopene decrease the oxidative stress and inflammation induced by iron in rats with colitis. J Gastroenterol 39, 514-519.

12. Bansal P, Gupta SK, Ojha SK, et al. (2006) Cardioprotective effect of lycopene in the experimental model of myocardial ischemia-reperfusion injury. Mol Cell Biochem 289, 1-9. 
13. Sepodes B, Maio R, Pinto R, et al. (2004) Tempol, an intracelullar free radical scavenger, reduces liver injury in hepatic ischemia-reperfusion in the rat. Transplant Proc 36, 849-853.

14. Bonta IL \& Bult H (1977) Effects of anti-inflammatory drugs on the carrageenin-induced hind paw inflammation of rats deprived of endogenous precursors of prostaglandins. Agents Actions Suppl 2, 77-83.

15. Wu Y, Zhou C, Song L, et al. (2006) Effect of total phenolics from Laggera alata on acute and chronic inflammation models. J Ethnopharmacol 108, 243-250.

16. Mota-Filipe H, Sepodes B, McDonald MC, et al. (2002) The novel PARP inhibitor 5-aminoisoquinolinone reduces the liver injury caused by ischemia and reperfusion in the rat. Med Sci Monit 8, BR444-BR453.

17. Moussa ME, Sarraf CE, Uemoto S, et al. (1996) Effect of total hepatic vascular exclusion during liver resection on hepatic ultrastructure. Liver Transpl Surg 2, 461-467.

18. Serracino-Inglott F, Habib NA \& Mathie RT (2001) Hepatic ischemia-reperfusion injury. Am J Surg 181, 160-166.

19. Colletti LM, Kunkel SL, Walz A, et al. (1996) The role of cytokine networks in the local liver injury following hepatic ischemia/reperfusion in the rat. Hepatology 23, 506-514.

20. Shirasugi N, Wakabayashi G, Shimazu M, et al. (1997) Up-regulation of oxygen-derived free radicals by interleukin-1 in hepatic ischemia/reperfusion injury. Transplantation 64, 1398-1403.

21. Ou B, Hampsch-Woodill M \& Prior RL (2001) Development and validation of an improved oxygen radical absorbance capacity assay using fluorescein as the fluorescent probe. $J$ Agric Food Chem 49, 4619-4626.

22. Cuzzocrea S, Riley DP, Caputi AP, et al. (2001) Antioxidant therapy: a new pharmacological approach in shock, inflammation, and ischemia/reperfusion injury. Pharmacol Rev 53, 135-159.

23. Di Rosa M, Giroud JP \& Willoughby DA (1971) Studies on the mediators of the acute inflammatory response induced in rats in different sites by carrageenan and turpentine. J Pathol $\mathbf{1 0 4}$ $15-29$.

24. Khattab MM (2006) Tempol, a membrane-permeable radical scavenger, attenuates peroxynitrite- and superoxide anionenhanced carrageenan-induced paw edema and hyperalgesia: a key role for superoxide anion. Eur J Pharmacol 548, 167-173.

25. Cuzzocrea S, Zingarelli B, Hake P, et al. (1998) Antiinflammatory effects of mercaptoethylguanidine, a combined inhibitor of nitric oxide synthase and peroxynitrite scavenger, in carrageenan-induced models of inflammation. Free Radic Biol Med 24, 450-459.

26. de Groot H \& Rauen U (2007) Ischemia-reperfusion injury: processes in pathogenetic networks: a review. Transplant Proc 39, 481-484.

27. Glantzounis GK, Salacinski HJ, Yang W, et al. (2005) The contemporary role of antioxidant therapy in attenuating liver ischemia-reperfusion injury: a review. Liver Transpl 11, 1031-1047.

28. Michael McClain R \& Bausch J (2003) Summary of safety studies conducted with synthetic lycopene. Regul Toxicol Pharmacol 37, 274-285. 